\title{
Clinical Trial Monitoring Visit Log
}

National Cancer Institute

\section{Source}

National Cancer Institute. Clinical Trial Monitoring Visit Log. NCI Thesaurus. Code C115536.

Records of the site monitoring visit dates and attendees in a clinical trial. 